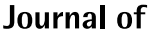 neurology \\ neurosurgery \&psychiatry
}

EDITORIAL

\section{Transcranial Doppler ultrasound}

The use of Doppler ultrasound to estimate blood flow velocity was described in $1960^{1}$ but it was only in the 1980 s that it was appreciated that sufficient ultrasound would pass through the skull to allow the detection of blood flow within the intracranial circulation. ${ }^{2}$ To achieve sufficient bone penetration low frequency ultrasound is used; $2 \mathrm{MHz}$ is conventional. This increases tissue penetration at the cost of poor spatial resolution. Therefore, even with more recent transcranial duplex scanners two dimensional B mode images are of low spatial resolution, and in adults the technique primarily provides useful information about blood flow velocity. Using various bone windows, where the barrier to the ultrasound is thinnest, it is possible to insonate the proximal middle, anterior, and posterior cerebral arteries, the distal internal carotid artery, and the intracranial vertebrobasilar system. ${ }^{3}$ Localisation is achieved by the use of pulsed Doppler ultrasound allowing determination of the depth of the insonated vessel. Recent colour flow duplex systems, which can display the Doppler information from a number of vessels simultaneously, improve the ease with which the intracranial vessels can be identified. They also allow angle correction and therefore more accurate blood velocity measurements.

Transcranial Doppler has a number of advantages as a method of evaluating cerebral haemodynamics. It is relatively cheap and non-invasive, allowing repeated measurements and continuous monitoring. It has a high temporal resolution making it ideal to study rapid changes in cerebral haemodynamics. On the negative side it relies on a trained technician, and in about $5 \%-10 \%$ of cases sufficient ultrasound cannot be transmitted through the skull to obtain a signal. In such cases the use of intravenous contrast agents, which contain stabilised microbubbles which pass into the arterial circulation and result in increased backscattering of ultrasound, may enable vessel identification. ${ }^{4}$ It should also be remembered that Doppler ultrasound measures velocity rather than flow, and therefore provides an estimation of cerebral blood flow only if vessel diameter remains unchanged. This assumption seems to be valid in most situations, but under certain conditions basal cerebral vessel diameter can change-for example, this seems to occur during pharmacological manipulation of the nitric oxide system. ${ }^{5}$

Much of the initial application of transcranial Doppler was in the detection of stenoses of the basal intracerebral arteries. Stenoses of greater than $50 \%$ or $60 \%$ can be identified by the accompanying increase in velocity through the narrowed artery. Transcranial Doppler ultrasound is routinely used in this clinical context in many countries. One obstacle to its more widespread implementation has been the lack of any specific treatment for intracranial stenoses. Elegant studies in acute stroke have shown that transcranial Doppler ultrasound can be used to demonstrate the time course of occlusion of the middle cerebral artery and subsequent recanalisation. ${ }^{6}$ An obvious application is in the identification of patients with persisting vessel occlusion for thrombolysis, therefore avoiding the use of thrombolytic agents in subjects who have spontaneously reperfused. However, current evidence of the length of the potential time window implies this would require the availability of transcranial Doppler ultrasound in the accident and emergency department. Furthermore, transcranial Doppler ultrasound may miss branch occlusions and such patients might benefit from thrombolysis. It is particularly suitable when repeated measurements are required. Two such contexts are subarachnoid haemorrhage and sickle cell disease. Transcranial Doppler ultrasound can demonstrate vasospasm after subarachnoid haemorrhage and is used by some neurosurgical units to guide management. ${ }^{7}$ Stroke is a common complication in sickle cell disease, particularly in children, and is often secondary to stenoses of the intracranial arteries. A recent trial showed that patients with intracranial stenoses detected by transcranial Doppler ultrasound, who were randomised to treatment with regular exchange transfusions, had a lower stroke rate than controls. ${ }^{8}$

The high temporal resolution of transcranial Doppler makes it ideal to study rapid changes in cerebral haemodynamics. One such application is the detection of impaired cerebrovascular haemodynamic reserve. Blood flow velocity in the middle cerebral artery is monitored before, and during, administration of either a mixture of carbon dioxide in air or the carbonic anhydrase inhibitor acetazolamide. Under such circumstances middle cerebral artery diameter remains constant. ${ }^{9}$ In normal subjects there is a marked increase in flow velocity of $50 \%$ or greater depending on the carbon dioxide concentration used. In a minority of patients with carotid occlusion or tight carotid stenosis, essentially those with poor collateral supply, carbon dioxide reactivity is markedly impaired. ${ }^{10}$ In patients with carotid occlusion and exhausted reserve the risk of stroke seems to be greatly increased. ${ }^{11}$ The technique is useful in identifying those hypertensive patients with carotid occlusion and markedly impaired haemodynamic reserve who may develop ischaemia when antihypertensive medication is instituted. It may also aid in guiding the need for revascularisation procedures in the small group of patients with carotid occlusion and haemodynamic stroke or transient ischaemic attack. In patients with carotid stenosis, rather than occlusion, an impaired carbon dioxide reactivity may 
also confer increased stroke risk, although there is less available data for this patient group. If larger natural history studies confirm this association, the technique may be useful in selecting those patients with asymptomatic carotid stenosis who might benefit from carotid endarterectomy. It may also aid in determining whether patients undergoing cardiopulmonary bypass who have asymptomatic carotid stenosis should undergo carotid endarterectomy.

Carbon dioxide reactivity provides an indirect estimate of "cerebral autoregulation", but more recently transcranial Doppler ultrasound has been used to estimate cerebral autoregulation directly. Existing methods of determining cerebral autoregulation usually require complex measurements of cerebral blood flow, and manipulation of systemic blood pressure with pharmacological agents. A direct estimate can be obtained by comparing the rate of rise of blood flow velocity in the middle cerebral artery with that of arterial blood pressure, after a sudden step reduction in systemic blood pressure. ${ }^{12}$ This is induced by the sudden deflation of leg cuffs previously inflated suprasystolically. Continuous arterial blood pressure measurements are usually achieved non-invasively by the use of a Finapress. In a normal subject flow velocity in the middle cerebral artery returns to baseline much more rapidly than blood pressure. This technique provides a unique tool to explore the importance of impaired dynamic autoregulation in the pathophysiology of cerebral ischaemia. Impaired autoregulation occurs in hypertension, and is thought to play a part in acute cerebral ischaemia. Using this technique impaired dynamic autoregulation has been shown in a subgroup of patients with carotid artery stenosis. ${ }^{13}$ It has also been used to investigate the normal pharmacology of cerebral autoregulation, and has demonstrated a potential role of nitric oxide in mediating this response. ${ }^{14}$

The non-invasive nature and high temporal resolution of transcranial Doppler ultrasound make it ideal for perioperative monitoring. It is widely used for monitoring during carotid endarterectomy. Indications for perioperative shunting vary markedly between different surgeons, but some use a reduction in middle cerebral artery velocity on cross clamping as an indication of the need for shunt insertion. Continuous monitoring throughout the operation can also give an indication of other inadvertent events such as shunt kinking.

Ultrasound offers the unique ability to detect emboli as they pass through the circulation. Emboli are seen as short duration high intensity signals due to the fact that more ultrasound is scattered and reflected at the embolus-blood interface, than is scattered by the surrounding blood. ${ }^{15}$ Transcranial Doppler has been used to detect presumed gaseous emboli during cardiopulmonary bypass for many years but it was only in 1990 that it was appreciated that solid (platelet and thrombus) emboli could also be detected. ${ }^{16}$ Some experimental studies have confirmed the sensitivity and specificity of the technique. ${ }^{17}$ Asymptomatic emboli have been detected in patients with a wide variety of potential embolic sources including carotid artery stenosis, intracranial stenoses, valvular heart disease, atrial fibrillation, recent myocardial infarction, and during and immediately after both carotid endarterectomy and angioplasty. ${ }^{15} \mathrm{In}$ many of these conditions the clinical relevance of these asymptomatic embolic signals remains uncertain, and large natural history studies are required. However, the technique offers exciting applications particularly in the identification of high risk patients for appropriate pharmacological and surgical intervention, in localisation of the embolic source in patients with multiple potential sources, and in monitoring the effectiveness of pharmacological therapies. Most studies have been performed in carotid artery stenosis. Asymptomatic cerebral emboli are surprisingly frequent even during recording periods as short as 1 hour, and are found in $20 \%-70 \%$ of patients with symptomatic stenosis and $5 \%-20 \%$ of patients with asymptomatic stenosis. Their presence correlates with known indicators of increased stroke risk; they are commoner soon after a transient ischaemic attack or stroke, in symptomatic stenosis compared with asymptomatic stenosis, and in the presence of plaque ulceration determined angiographically or histologically. ${ }^{15}$ In one patient they were seen to pass through the middle cerebral artery at the time of onset of both neurological deficit and detection of retinal emboli. ${ }^{18}$ In a small prospective study in patients with asymptomatic carotid stenosis they were predictive of future stroke and risk for transient ischaemic attack. ${ }^{19}$ If this finding is confirmed in larger studies transcranial Doppler ultrasound may prove a useful technique in identifying which patients with asymptomatic carotid stenosis will benefit most from endarterectomy. Operating on all patients with greater than $60 \%$ or $70 \%$ stenosis is of questionable benefit and markers of high risk are needed..$^{20}$ An additional exciting use of embolic signal monitoring is its potential to evaluate new antiplatelet agents. Currently, such agents have to be evaluated in animal models, or by ex vivo assays, before large clinical trials. Neither are truly representative of the situation in humans. Due to their much greater frequency compared with transient ischaemic attack and stroke, monitoring for embolic signals offers the potential to evaluate new agents using relatively few patients. This use has been demonstrated after carotid endarterectomy during which removal of the endothelium and inner media occurs creating a potent thrombogenic surface. Embolic signals are frequent after the operation, and a high frequency predicts subsequent stroke and risk of transient aschaemic attack. ${ }^{21}$ This situation has recently been used to show the effectiveness of a potentially platelet specific nitric oxide donor S-nitrosoglutathione. ${ }^{22}$ There was a significant reduction in embolisation in the group treated with S-nitrosoglutathione, despite the use of aspirin and heparin in both groups. The potential of the technique is shown by the fact that a sample size of only 12 was required in each group. These findings will need to be confirmed in clinical studies using the end point of stroke, but the technique may allow optimal antithromboembolic regimens to be determined before large expensive clinical trials.

HUGH S MARKUS

Department of Clinical Neurosciences, King's College School of Medicine and Dentistry and the Institute of Psychiatry

Correspondence to: Dr Hugh Markus, Department of Clinical Neurosciences, Institute of Psychiatry, De Crespigny Park, London SE5 8AF, UK. Telephone 0044171 3465174; fax 0044171 3465186; email h.markus@iop.bpmf.ac.uk.

1 Satomura S. Kaneko Z. Ultrasonic blood rheograph. Proceedings of the 3rd International Conference on Medical Electronics. 1960:254-8.

2 Aaslid R. Markwalder T-M, Nornes H. Non-invasive transcranial Doppler ultrasound recording of flow velocity in basal cerebral arteries. $\mathcal{F}$ Neurosurg 1982;50:570-7.

3 Fujioka K A, Douville C M. Anatomy and freehand examination techniques. In: Newell DW, Aaslid R. eds. Transcranial Doppler. New York: Raven Press, 1992.

4 Ries F. Echocontrast agents in transcranial Doppler sonography. In: Tegeler CH, Babikian VL, Gomez CR, eds. Neurosonology. St Louis: Mosby, 1995: 221-8.

5 Dahl A, Russell D, Nyberg Hansen R, et al. Effect of nitroglycerin on cerebral circulation measured by transcranial Doppler and SPECT. Stroke $1989 ; 20: 1733-6$

6 Ringlestein EB, Biniek R. Weiller C, et al. Type and extent of hemispheric brain infarctions and clinical outcome in early and delayed middel cerebral artery recanalization. Neurology 1992;42:289-98.

7 Sloan MA. Transcranial Doppler monitoring of vasospasm after subarachnoid haemorrhage. In: Tegeler $\mathrm{CH}$, Babikian VL, Gomez CR, eds. Neurosonology. St Louis: Mosby, 1995:156-71.

8 Adams RJ, McKie VC, Hsu L, et al. Prevention of first stroke by transfusions in children with sickle cell anaemia and abnormal results on transcranial Doppler ultrasonography. $N$ Engl f Med 1998;339:5-11.

9 Huber P, Handa J. Effect of contrast material, hypercapnia, hyperventilation, hypertonic glucose and papaverine on the diameter of cerebral arteries. Invest Radiol 1967;2:17-32. 
10 Ringelstein EB, Sievers C, Ecker S, et al. Non-invasive assessment of $\mathrm{CO}_{2}$ induced cerebral vasomotor response in normal individuals and patients . Stroke 1988;19:963-9.

11 Kleiser B. Widder B. Course of carotid artery occlusions with impaired carbon dioxide reactivity. Stroke 1992;23:171-4.

12 Newell DW, Aaslid R, Lam AM, et al. Comparison of flow and velocity during dynamic autoregulation testing in humans. Stroke 1994;25:793-7.

13 White RP, Markus HS. Non-invasive determination of impaired dynamic cerebral autoregulation in carotid artery stenosis. Stroke 1997;28:1340-4.

14 White RP, Vallance P, Markus HS. Cerebral autoregulation in man is nitric oxide dependent. Cerebrovas Dis 1998;8(suppl 4):100

15 Markus HS, Harrison MJ. Microembolic signal detection using ultrasound. Stroke 1995;26:1517-9.

16 Spencer MP, Thomas GI, Nicholls SC, et al. Detection of middle cerebral artery emboli during carotid endarterectomy using transcranial Doppler ultrasonography. Stroke. 1990;21:415-23.
17 Markus HS, Tegeler C. Experimental aspects of high-intensity transient signals in the detection of emboli. F Clin Ultrasound 1995;23:81-7.

18 Khaffaf N, Karnik R, Winkler WB, et al. Embolic stroke by compression maneuver during transcranial Doppler sonography. Stroke 1994;25:1056-

19 Siebler M, Nachtmann A, Sitzer M, et al. Steinmetz H. Cerebral microembolism and the risk of ischaemia in asymptomatic high-grade internal carotid artery ischaemia. Stroke 1995;26:2184-6.

20 Warlow C. Endarterectomy for asymptomatic carotid stenosis? Lancet 1995; 345:1254.

21 Levi CR, O'Malley HM, Fell G, et al. Transcranial Doppler-detected cerebral microembolism following carotid endarterectomy: high microembolic signal laoads predict post-operative cerebral ischaemia. Brain 1997;120:621-9.

22 Molloy J, Martin JF, Baskerville PA, et al. S-nitrosoglutathione reduces the rate of embolisation in humans. Circulation 1998;98:1372-5.

\section{NEUROLOGICAL STAMP}

\section{Sir William Osler (1849-1919)}

"William Osler, the younger son in a family of 9 , was born on 12 July 1849 in a parsonage at Bond Head, Tecumseth County near the edge of the wilderness in what was Upper Canada. How this came about, as to place, time and circumstances, needs telling from the very beginning." It was with these words that Harvey Cushing began The Life of Sir William Osler, the autobiography for which he was awarded the Pulitzer Prize in 1925. Osler was at various times Professor Medicine at McGill (at the age of 26), the University of Pennsylvania, John Hopkins, and finally Regius Professor of Medicine at Oxford. He was one of the first to describe platelets (1876) and wrote monographs on abdominal tumours (1895) and malignant endocarditis. (1908). Osler described polycythemia rubra vera (1903), but later learned of and insisted on the priority of Vaquez. The disorder is also known as Vaquez-Osler disease or Osler disease. Hereditary telangiectasia with recurrent haemorrhages described by Osler in 1908 is also known as Osler disease. Osler also drew attention to Osler's nodes of endocarditis.

At Baltimore he soon became a commanding figure in American medicine. There he pioneered new teaching methods in medicine, including ward tuition and an emphasis on laboratory techniques. His close friends included Mitchell and Cushing in America as well as most of the leading English neurologists including Ferrier, Horsley, Sherrington and Gowers, whom Osler referred to as "that brilliant ornament of British medicine". His monographs on Chorea and Choreiform Affections (1894) and The Cerebral Palsies of Children (1889) were written from his experiences in Philadelphia where he was closely associated with Mitchell. In The Cardiac Relations of Chorea he emphasised the extraordinary frequency with which mitral valvitis "lays the foundation of organic heart disease". Other neurological contributions were papers on cerebral haemorrhage, concussion, brain tumours, aneurysms, cerebral emboli, infantile paralysis, meningitis, and cerebrospinal fever.

Osler's Principles and Practice of Medicine was the leading English textbook of medicine at the time. In this he wrote an extensive section on diseases of the nervous system. His contributions placed Osler among the foremost neurologists of the period, and he was one of the greatest physicians of his time. The Principles and Practice of Medicine which appeared in 1892 went through eight additions during the author's lifetime and was translated into French, German, Spanish, and Chinese. He was editor of Modern Medicine, (1910) and editor and founder of the Quarterly fournal of Medicine, (1908). His writings, like his personality, had an elusive charm. Osler was an inveterate collector of books and bequeathed the bulk of his library to his alma mater, McGill University in Montreal. Although Osler did not make profound or fundamental discoveries, there was no other of his day who in his life and teaching radiated such an inspiration to fellow physicians. Osler had one son, Revere, named after his great great grandfather, Paul Revere. Paul Revere's midnight ride to Lexington and Concord (April 1775) to warn the people of the approach of British troops was rendered famous by Longfellow's poem The Midnight Ride of Paul Revere. The death of

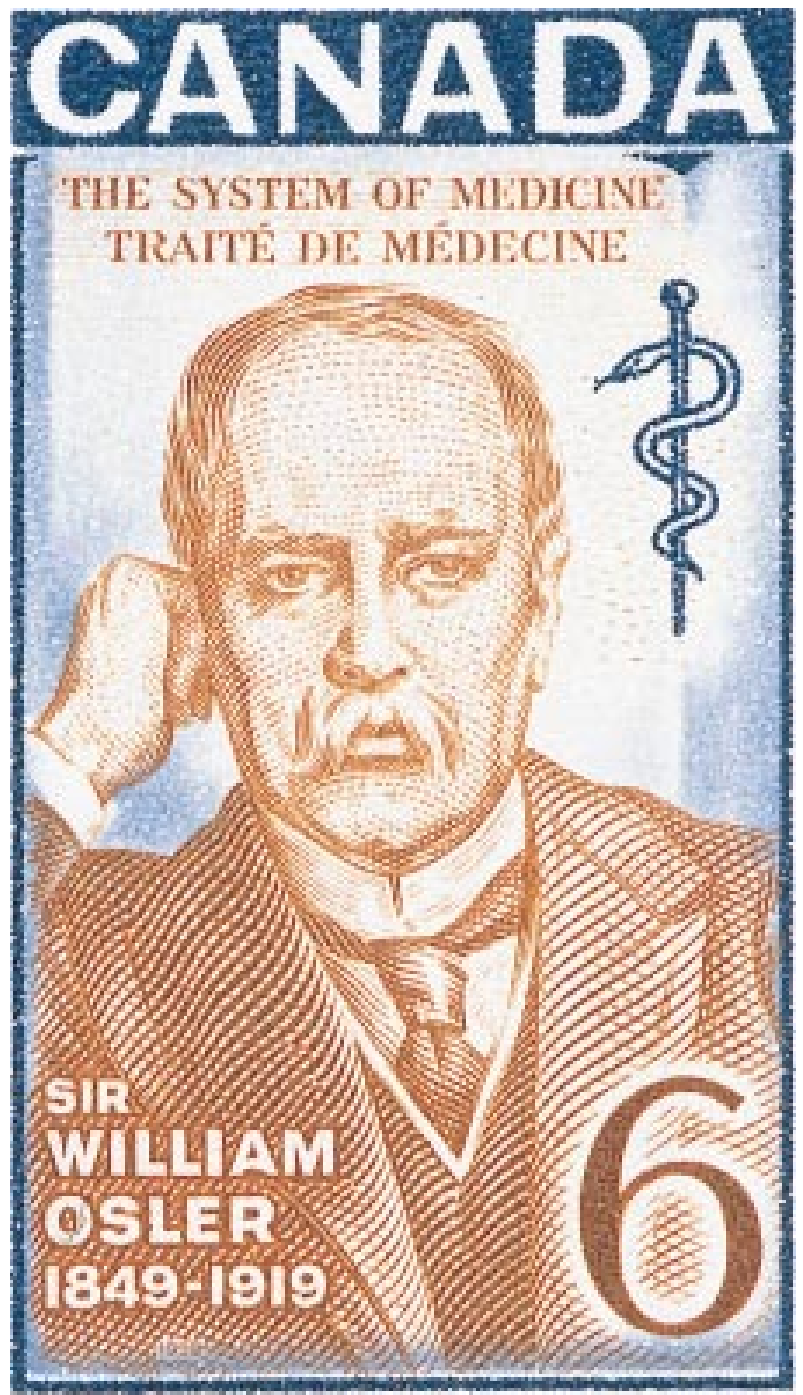

his son in the first world war was a great loss. Osler was created Baronet in 1911 and received honorary degrees in America and across the Atlantic from McGill, Toronto, Harvard, John Hopkins, Edinburgh, Oxford, Leeds, Christiania, Dublin, Durham, and Cambridge.

He was honoured philatelically by Canada in 1969 on the 50th Anniversary of his death (Stanley Gibbons 637, Scott 495) 